\title{
A 'new’ Village Sign Language: structural properties of LSOR in Puerto Rico?
}

\author{
Elena Benedicto (Purdue University) \\ ebenedi@purdue.edu \\ Marina Martínez-Cora (Universidad de Puerto Rico-Río Piedras) \\ yolanda.rivera5@upr.edu \\ Yolanda Rivera-Castillo (Universidad de Puerto Rico-Río Piedras) \\ marina.martinez@upr.edu
}

\begin{abstract}
In this preliminary analysis, we describe the use of the signing space in morphosyntactic processes in Orocovis Sign Language (LSOR), a Village Sign Language shared as the medium of communication between Deaf and hearing members of a rural community in Puerto Rico. It has been passed on from generation to generation, and exhibits an extended and absolute use of the signing space, and grammatical features like agreement, aspectual marking, and classifiers. These preliminary results contribute to shape the emerging typological complexity in the area of Village Sign Languages.
\end{abstract}

Keywords: Village Sign Language, signing space, agreement, aspect, classifiers

\section{Introduction and goals}

In this paper we address the properties of an up-to-date unidentified Village Sign language (VSL) in the community of Orocovis in Puerto Rico, LSOR (Lengua de Señas de Orocovis). The language is used by a cohesive community of Deaf and hearing signers, located in an inland semi-rural area of Puerto Rico. It is a different sign language from the one commonly used in Puerto Rico, PRSL (Puerto Rico Sign Language), which in turn is different from ASL (Martínez-Cora 2019).

Our goals for this project are twofold: on the one hand, to evaluate LSOR's structural linguistic properties; on the other hand, to assess those properties within the range of properties previously observed in other VSLs, so as to ascertain the status of LSOR with respect to other VSLs, and of VSLs in general with respect to SLs more widely.

For that, we examine recurrent patterns found in our dataset, associated with: (i) the use of (signing) space; (ii) agreement; (iii) aspect; and (iv) classifiers. These are the structural areas of inquiry that have proven to be of interest in the characterization of VSLs in the past, as we will discuss in the following section. 


\section{Background}

\subsection{About the notion of VSLs}

VSLs have been attracting increasing attention in the last couple of decades for a variety of reasons. One of them is the fact that they can shed light on understanding the formation processes of sign languages, the conditions that make a language a language; and another is for their role in understanding the typology of sign languages, the variation found within them, and the typological contrast between languages in the signed modality and in the spoken modality (U. Zeshan 2008; Zeshan and Vos 2012).

The conditions that favor the existence of VSLs have also had an effect in how they are referred to; in addition to Village SL, they have been addressed as rural SLs, in contraposition to larger, urban SLs (e.g., De Vos and Nyst 2018; De Vos and Pfau 2015; De Vos 2012), and as Shared SL (e.g., Nyst 2012) as they are used by both deaf and hearing individuals. In identifying the factors that contribute to their existence, researchers have pinpointed conditions related to the number of deaf individuals within the community, usually higher than in other communities, but which can range from single digits to several tens, reaching the hundreds in some cases (De Vos and Pfau 2015); the number of hearing signers, from hundreds to more than a thousand, and the ratio of deaf/hearing signers; and the time depth of the SL (De Vos and Nyst 2018), which can also vary notably from one case to another.

VSLs indeed are not a homogeneous group, as the different studies keep showing (see De Vos and Pfau 2015) and there is a large range of variation in the conditions mentioned above. In our own thinking (about LSOR in particular), we consider the high density of deaf individuals, the robust participation of hearing signers (who might be considered bilingual Spanish/LSOR), and a linguistic ecosystem that those two conditions create that provides a sufficient and healthy environment for everyday language use to arise, both in terms of numbers (e.g., the potential for a deaf signer to be able to fully interact in SL with any person in the community) and in terms of diversity of experience within the community (so that every and any interaction a deaf signer has is a quality SL interaction). In that sense, we prefer the term Village because it better reflects the texture, density and strength of the community weaving (to borrow a metaphor from Benedicto and Salomón 2017, dealing with the density and robustness of cultural interaction in language maintenance). In that respect, albeit not entirely in the same sense, we get close to the 'ecology' perspective found in Nyst (2012).

Under this notion of community (village), it doesn't matter whether the community is in a rural area or in an urban neighborhood; it does not matter so much that the SL is shared with hearing members, but how it is shared: what matters is the quality of that interaction, the tightness of the community wherever it is, that allows for a true language community to arise in the most normal way (that is, the way that allows for full language acquisition across generations, supported by family and neighborhood, and for a healthy community of language use).

Ironically, from the perspective of survival of such VSLs, access to a formal education system (and, thus, a national SL) may be a hindrance to the maintenance of the VSLs (see the ambivalence expressed in the way certain news portray the community, e.g., Gonzalo 2014; see also Section 2.3 below).

\subsection{VSLs in theoretical linguistic terms}

In terms of the linguistic characterization of VSLs, certain parameters have been considered to be of relevance, in particular at 3 levels: the lexical system (color systems, kinship, and 
numeral systems); the use of space for referential purposes (whether absolute or abstract); and at the morphosyntactic level, the use of space in, e.g., agreement and the use of a variety of classifiers in locative and motion predicates. A good recent summary of these and other topics can be found in De Vos and Zeshan (2012), Nyst (2012), and De Vos and Pfau (2015).

In our first approximation to characterize LSOR, and given the state of the art with respect to VSLs, we chose to examine grammatical aspects of the language concerning the use of space and any evidence of inflectional systems in the verbal domain. We will thus here concentrate on providing an overview of the main issues under discussion in those areas.

With respect to the use of space, the literature has made a distinction between the use of absolute and abstract (metaphoric, space-restricted) locations. The first one makes use of actual locations in the space widely set around the signer, while the second one sets arbitrary locations, in a reduced signing space typically in front of the signer. Whereas the use of an absolute pointing system has been observed as a primary system in most VSLs, this is not a homogeneous property: De Vos and Pfau (2015) state, citing Schuit (2013), that Inuit SL has been documented to have both absolute and abstract loci. In terms of numbers of deaf signers, Inuit SL has the same number of signers as, say, Kata Kolok, which does not have abstract pointing (De Vos 2012). So, VSLs are not restricted only to an absolute use of space, and their specific use of space is not linked to the number of deaf signers.

Other features that have been explored as potentially characterizing VSLs are the use of agreement and the use of classifiers. Here too, variation has been observed: Zeshan (2010) discusses that Adamorobe SL has agreement (directional verbs, in her terms) whereas Kata Kolok does not; and that whereas Adamorobe SL does not show (whole entity) classifiers, Kata Kolok does have them. So, no particular pattern seems to arise there either.

In fact, De Vos and Pfau (2015) and Nyst (2012) consider an interesting hypothesis linking those three properties: that the particular use of space (whether absolute or abstract) determines whether a language will be able to use space modulations such as agreement and classifiers. If a language has absolute pointing, it is hypothesized, it will not be able to engage in agreement (assuming, following De Vos 2012, that 'deictic pointing signs do not license spatial modification') or classifiers (Nyst 2007). However, they find that two VSLs, Inuit SL and Yolngu SL, both have absolute pointing and use such locations for the spatial modification of verbs and for classifier predicates. So, in that respect too, VSLs manifest a variable behavior.

In summary, VSLs do not seem to show a homogeneous pattern of behavior, and thus they do not seem to form a typological class, as already recognized in De Vos and Pfau (2015). From a typological point of view, large urban SLs have both abstract pointing and agreement and classifier structures. What VSLs have shown is that neither of those factors are universal features of SLs in general (since some, but not all VSLs lack them).

Given this state of affairs, then, we set out to explore the use of space in LSOR: to see if it has an absolute use of spatial locations, and to see if that system is engaged in agreement (directional) verbal forms and classifier verbal forms. We also want to explore the potential range of classifier types (whole entity, handling, among others), as it is part of the discussion about VSLs. Finally, a last feature in the morphosyntactic realm is the use of aspectual inflection in the verbal system. We will address these points in Section 4.

\subsection{The linguistic situation on the island}

Most signers in Puerto Rico, including some young Orocovis signers, use PRSL. MartínezCora (2019), who has described some PRSL features, including non-manual markers, de- 
scribe it as mainly urban and co-existing with ASL in some formal settings. LSOR is also in contact with Spanish, the main spoken language in the island (Martínez-Cora 2018).

The relation between all those languages, both spoken and signed, is a hierarchical one, involving deeply rooted issues of power. Society tends to categorize languages into first-, second- and third-class languages (De Meulder et al. 2019; Eckert and Rickford 2002; Woodbury, n.d.; Trovato 2013). A first-class language is one with the most prestige, such as those that belong to past colonizing powers (English, Spanish, etc.); while second-class languages are indigenous languages that may have developed a writing system; and the sign languages of the world can be considered third-class languages since they do not have writing systems and, for this reason, may not be considered natural linguistic systems in certain circles.

The history behind the emergence, development and transmission of the different sign languages in Puerto Rico is still under research. ${ }^{1}$ However, this transmission is tied to various factors. One of them is the establishment of the first Deaf school in Puerto Rico, "Colegio San Gabriel para Sordos" (http://csg.edu.pr). Due to the complex political situation on the island, there are issues with access to government services by members of the Deaf community. For example, due to the need for qualified interpreters, the local government decided to send various groups of people to be trained in ASL in the USA. These first interpreters have described difficulties when trying to communicate with Deaf clients on the island. This has happened because the sign language that the Deaf knew, was not the same language taught to the interpreters (Williams and Parks 2012; Annarino, Aponte-Samalot, and Quinto-Pozos 2014). According to previous research, one of the sign languages used in Puerto Rico is an ASL dialect (Williams and Parks 2012), but it should be noted that the authors have witnessed how Deaf individuals from the continental US point out differences in the grammar used by fellow interpreters in Puerto Rico and by the Deaf community.

On the other hand, the role of dominant sign languages in the displacement and endangerment of local sign languages cannot be underestimated (Braithwaite 2019). Language contact is common in Deaf communities (Quinto-Pozos and Adam 2015), including contact with spoken languages (LeMaster and Monaghan 2005, 144). Contact with spoken languages triggers the creation of other myths, such as the widespread belief that PRSL is a form of Spanish (which the most commonly spoken language in Puerto Rico, according to the Census Bureau 2005).

PRSL has not been recognized because it lacks the prestige that ASL has in the hearing community. There is a direct relation between the political situation on the island as a territory of the US and the role of ASL on the island. The US, as a national power, has languages, like English and ASL, which have become dominant languages; meanwhile, minority languages like PRSL are not recognized as such. The local Deaf community has argued in favor of the recognition of PRSL as the sign language of the island but the government has failed to do it. The reason for their actions is based on the status given to ASL as a language of power.

\subsection{The LSOR Deaf community}

The LSOR Deaf community includes between 25 and 50 extended mostly black Caribbean families, in a semi-rural setting. Though there is indeed a high concentration of D/deaf individuals, the families also include hearing members who also use LSOR as the community language. LSOR is acquired from birth within the family and the neighborhood at large. It is unclear as of yet how many generations of signers there can be, but, at least at this point,

1. A recent paper by Quiñones was presented at the SSML-2 in August 2021, about the sociolinguistic status of PSRL. However, the authors have not been able to access it beyond the presentation. 
four generations of adults can be identified. The patterns reported below, however, are those consistently shared by all members of the signing community.

\section{Methods}

Results reported constitute part of a larger documentation project for LSOR. The data described so far for the bigger project include interviews, both at the individual level and in family groups. Further work will include: [1] Focus Groups (by gender, age and neighborhood); and [2] targeted data collection (for motion predicates). We report on the first subset (individual and family interviews) for a multigenerational family of Deaf individuals of different ages.

Team members visited the community on five occasions. The first three visits included introductions and description of project activities. Recordings took place at the home of the family. We conducted interviews in Puerto Rican Sign Language (PRSL), with the help of one project researcher.. Then, communication in LSOR was conducted via young signers that translated from PRSL to LSOR. Participants were instructed to answer in LSOR, although some code-switching happened during portions of the interview. Since younger members of the community are multilingual (PRSL and LSOR), they interpreted instructions for older members of the community who are monolingual in LSOR.

Two research project members placed cameras from two different angles (frontal and sideways). A research team member directed the first set of interviews, while the following ones were directed by younger members of the family. Open questions were asked about their family history, among other general topics, and follow up questions were formulated as the conversation progressed. Some questions required indicating locations, describing agricultural work, and asking for numbers (family size, for example). After recordings were completed, the team uploaded data to a safe institutional server. Recordings were segmented, integrated into ELAN and analyzed to identify features related to the use of signing space and the other grammatical features initially selected for analysis. Further work to complete a comprehensive transcription and coding is ongoing.

\section{Results and analysis}

In this section, we take up the structural elements we set out to explore after the discussion of structural patterns found in VSLs (Section 2.2) and explain the identification of patterns that we have documented in our dataset, with respect to the four areas mentioned in Section 1: (i) the use of space; (ii) agreement; (iii) aspect; and (iv) classifiers. In Section 5, we will evaluate these results with respect to properties observed in other VSLs and in SLs in general.

\subsection{LSOR's Signing Space}

We address first the use of space since it is one of the features regularly observed in the study of VSLs (see Section 2.2) and because it will become relevant for the subsequent subsections, in particular, those dealing with agreement and classifiers.

First, we have observed that LSOR uses an extended signing space, which means that it is not restricted to the space in front of the signer: it can use the space behind the signer, and it can also use body locations like the legs. This extended signing space has been observed in many VSLs. 
Secondly, LSOR also shows an extensive use of absolute pointing. As mentioned in Section 2.2, absolute pointing involves pointing towards real space loci. In our dataset, we can observe that LSOR signers, when using space to identify the locations of referents, can use the pointing sign IX directed towards an actual location, as in (1) where the IX sign points towards a location in the back-center $\left(\mathrm{IX}_{\mathrm{bk}-\mathrm{ctr}}\right)^{2}$ behind the signer, or in (2) where the signer points towards a location in the front-left-up ( $\mathrm{IX}_{\text {frt-lft-up }}$ ), to indicate where the referent in question is (or was, at some point) located.

(1) DAD 1 HOUSE IX bk-ctr, HOUSE

[LSOR_T1_PPMM.06:24]

'My dad had a house over there.'

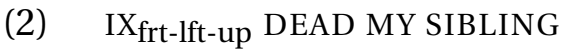

[LSOR_T1_PPMM.08:28]

'He (over there, at the front-left-up) died, my sibling.'

We also observed the use of absolute pointing for locations that are not visually accessible from the location of the conversation (as is the case in some of these examples). These absolute locations can be used in a variety of grammatical referential purposes:

a. They can be used as referential index locations for an argument associated to that specific location; in (3) below, the location back-center ( $\mathrm{IX}_{\mathrm{bk}-\mathrm{ctr}}$ ) is used as a referential index for the identification of the subject argument, associated with that particular location:

(3) DEAF IX bk-ctr MY SIBLING

[LSOR_T1_PPMM.07:20]

'He (over there, back-center) was deaf, my sibling.'

b. They can be used as transient sentence-specific actual locations, occupied by participants at the specific time of the utterance. In (4), the referent of $\mathrm{IX}_{\text {frt-lft-up }}$ is at the front-left-up position across from the signer, at that particular moment, but she was coming from another location, and was moving to another location in the room; the same can be observed in example (6) later:

(4) IX2 LOOK-AT IX frt-lft-up

[LSOR_T1_PPMM.06:36]

'You look at her [now at the front-left-up position].'

c. Finally, they can also be used symbolically, as a grammatical referential index for an argument not currently (at time of utterance) at that location, as in (5) or in (2) (above). In (5), the referent of $\mathrm{IX}_{\mathrm{lft}-\mathrm{ctr}}$, associated at the left-center location, is clearly not there anymore since he left. In the case of (2), the actual referent is not alive anymore. The association of the argument to the location is thus abstract, symbolic.

(5) IX $\mathrm{X}_{\text {Ift-ctr } \ldots \text { FLEW-AWAY }}$ rgt-up

[LSOR_T1_PPMM.02:22]

'He (over there, at left-center) flew away (towards right-up).'

2. Transcriptions use IX for the index pointing sign, with 1 indicating $1^{\text {st }}$ person (directed at self), 2 indicating $2^{\text {nd }}$ person (directed at interlocutor). For other directionalities, the following system is used, in any necessary combination: bk - back, frt -front; ctr -center, lft - left, rgt - right; up - up, dwn - down. If the coding of the handshape is relevant (as with classifiers), alphabet letters are used (one for each hand) with a "+" sign used to link the motion the handshapes engage in (see, e.g., example in (6)). 
Both a. and c. are cases where the location is not directly visible from where the signer is standing; they are thus cases of distal (non-visible) deixis. ${ }^{3}$

As we see from the cases above, even though the space location is absolute, it can and does enter into a variety of grammatical processes. Some of these grammatical processes will be further examined in the subsections that follow. Participation in these grammatical processes is interesting because the presence of an absolute system has been hypothesized as a reason for the lack of both agreement and classifier constructions in other VSL (cf. Nyst 2012 for AdaSL and De Vos 2012 for Kata Kolok). We retake this issue in Section 5.

\subsection{Agreement}

LSOR shows evidence of use of agreement devices. For that, it uses any spatial location that has been previously set. Once a spatial location has been set in any of the ways stated in Section 4.1 above, that spatial location can be widely used as agreement locations for verbs. The example in (4) was a case in point. In (6), also, the predicate (C-C+PUT 'fit hearing aids') is articulated sequentially at each of the locations where the daughters of the signer are standing (at the point of the utterance, therefore a case of transient sentence-specific actual location):

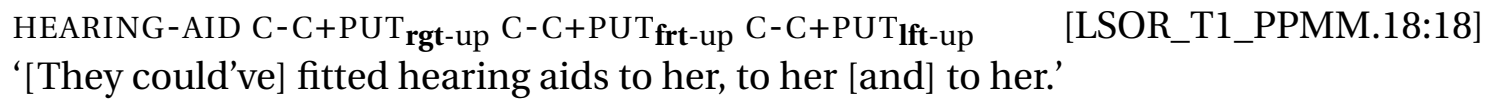

Notice that this means that absolute locations can be used for grammatical purposes, that is, they enter the grammatical system of the language.

\subsection{Aspect}

The presence or absence of inflectional morphology has been suggested as an indicator of the completeness (or lack thereof) of the grammatical systems of VSLs. In that sense, we wanted to observe if there was other inflectional morphology identified in LSOR. Indeed, verbs in LSOR can also show aspectual inflection. This takes the shape of a reduplicative movement (usually three times) along an arc-shape. The meaning associated with it is that of a continuous activity (not mere repetition), an unbound event similar to the Spanish imperfective. See (7) and (8), as examples:

$$
\text { SOW-SOW-SOW }
$$

[LSOR_T1_PPMM.15:06]

'(In those times) I was sowing.'

(8) HOE HOE HOE

[LSOR_T1_PPMM.15:32]

$$
\text { '[First, ... then] I hoed the ground, [then ... ].' }
$$

3. This type of referential deixis is also reminiscent of some systems found in (spoken) languages throughout Central America, where anchoring referential devices refer to locations not in existence anymore. In Nicaragua and Costa Rica, for instance, it is common to indicate the location of an entity as this: 'From where the Red Cross Hospital was, 2 blocks South', where the Red Cross Hospital has not been in existence for decades. This occurs in both urban and rural areas. 


\subsection{Classifiers}

The presence of classifier ${ }^{4}$ predicates in LSOR is one more piece of evidence of the grammatical complexity of the language. It also bears on the discussion whether the existence or absence of classifiers may be linked to an absolute use of space.

We can observe two properties in LSOR: one, the grammatical use of space, including absolute space, to articulate the classifier predicate; and two, a variety of types of classifier handshapes. With respect to the use of space, once a location has been established in previous discourse (in any of the ways described in Section 4.1), that location can be used as a location for the articulation of a classifier predicate. Notice that all the locations identified in 4.1 are absolute locations.

An example of that is the case of (9), where a location was set up, in previous discourse, in the back left up (bk-lft-up) of the signer and is now being used to utter a whole entity classifier to denote a hill:

$$
\begin{aligned}
& \text { IX } X_{\text {bk-lft-up }} . . . \text { B-B+EXTEND-UP } \\
& \text { 'That hill extends all the way up.' }
\end{aligned}
$$

[LSOR_T1_PPMM.07:22]

Beyond the whole entity classifier of (9), handling and instrumental classifiers can also be observed in (10) and (11) below, respectively.

(10) $5_{\text {closed }}-5_{\text {closed }>\text { open }}+$ SOW-SOW-SOW

[LSOR_T1_PPMM.15:06]

'(In those times) I was sowing.'

CUT.GRASS.WITH.TRIMMER

[LSOR_T1_SH.7:15]

'(He used to) cut grass with a trimmer.'

Thus, not only the presence of classifiers is established in LSOR, but the full range of classifier types seem to be documented too. This again contrasts with the lack or paucity of classifiers that has been documented in other, though not all, VSLs (Nyst 2012).

\section{Conclusions and future research}

In Sections 2.1 and 2.2 we established some of the main points of research about VSLs, in particular those (the use of signing space, agreement, classifiers and evidence of other inflectional phenomena like aspect) that have led the discussion about the position of VSLs within the typology of SLs and language in general.

From the phenomena described in Section 4 for LSOR, we can establish the generalizations in (12):

(12) Structural/Grammatical generalizations about LSOR

1. like large urban Deaf populations / unlike other VSLs, LSOR shows extensive use of (functional) morphology in

4. Based on the systematization in Benedicto and Brentari (2004) which follows Engberg-Pedersen (1993) classification, we determine that a particular predicate constitutes a classifier based on two properties: the handshape alternates with other handshapes in a paradigm of sorts (that is, the handshape behaves as a morpheme) and the predicate is anchored in space. The number of alternating handshapes may be small or large, what matters is the possibility of alternation. In some SLs, classifiers cannot inflect for aspect, but in some others they can; so this does not constitute an inherent property of classifiers. 
(a) agreement

(b) classifiers and

(c) inflectional aspect

2. unlike most large urban Deaf populations / like other VSLs, LSOR shows a use of the signing space that includes

(a) absolute pointing and

(b) a larger signing space.

This picture contributes to sharpen the complexity other researchers have already begun to identify in the field of VSLs, confirming that they do not form a homogeneous group. In particular, it seems to confirm that the specific status and use of the signing space (whether extended or restricted; whether absolute or abstract), does not determine its availability for its use as locations in agreement and classifier constructions. The table below shows a selection of VSLs from an overview of the available literature (e.g., Zeshan 2010; Nyst 2012; De Vos and Zeshan 2012; De Vos and Pfau 2015) about the behavior of relevant VSLs with respect to these phenomena and the position of LSOR among them (Table 1$).^{5}$

Table 1: VSLs and (non-)correlations for agreement, classifiers and signing space

\begin{tabular}{l|l|l|l|l} 
& \multicolumn{2}{|l|}{ (Signing) Space } & Grammatical Use & CLS \\
Language & Absolute & Abstract & AGR (directional) & - \\
\hline \hline AdaSL & + & & + & + \\
\hline KataKolok & + & - & - & + \\
\hline InuitSL & + & + & + (abstract and absolute) & + \\
\hline YolnguSL & + & + & $\begin{array}{l}+ \text { (absolute) } \\
- \text { (abstract) }\end{array}$ & \\
\hline LSOR & + & & + & + \\
\hline
\end{tabular}

Crucially, what we see is that InuitSL has agreement (directional Vs) with both absolute and abstract use of signing space) and YolnguSL has agreement (directional Vs) with only absolute use (De Vos and Pfau 2015, citing Schuit 2013 and Bauer 2014, respectively). LSOR thus patterns with these VSLs. More generally, this complex picture also seems to indicate that there may be a range of independent interacting parameters that determine the actual characteristics of any given VSL. What those parameters may be is a matter for further comparative investigation.

Finally, there is still a range of comparative issues that research on LSOR will need to address. A first one would be the matter of generational depth: what would be the relevance of LSOR's (relatively old) age for the observed properties? The matter of age has been addressed in the literature (e.g., De Vos and Nyst 2018) but a complete cohesive picture has not yet emerged. The point is how many generations of signers are needed for a grammatical system to arise that includes the phenomena in Table 1; but a deeper question might even be whether the presence/absence of these grammatical elements is at all an indicator of a fully developed grammar (or whether that presence/absence is just part of a typological divide). A second point would be the role of hearing signers in the community in determining the structural properties observed in LSOR: Is their signing structurally different? Does their spoken language affect LSOR? What is the ratio of deaf/hearing signers? These are questions

5. The cells with no content are those for which we haven't been able to establish the content with certainty. 
that will need to be addressed in the future. Finally, a third point would address the potential contact effects that more well- and widely-established SLs (such as PRSL) could have on the structural properties of LSOR.

\section{Acknowledgments}

We would like to thank the members of the Orocovis signing community who collaborated with our research in 2019. Also, we thank Purdue University (through a Global Synergy Grant Program) and the University of Puerto Rico (through its PEAF and UPRRP programs, with time release for research and research assistantship funding) for making it possible to conduct this research.

\section{References}

Annarino, Pauline, Myrelis Aponte-Samalot, and David Quinto-Pozos, eds. 2014. Hacia las Prácticas Efectivas: Interpretación en Situaciones Influenciadas por el Español. NCIEC.

Bauer, Anastasia. 2014. The use of signing space in a shared sign language of Australia. Sign language typology 5. Boston: De Gruyter Mouton.

Benedicto, Elena, and Diane Brentari. 2004. "Where did all the arguments go?: Argumentchanging properties of classifiers in ASL." Natural Language \& Linguistic Theory 22 (4): 743-810.

Benedicto, Elena, and Elizabeth Salomón. 2017. "Strong sense of identity as part of cultural well-being: documenting plant knowledge.” In Vital Voices: Linking Language \& Wellbeing. Presented at the 5th ICLDC (International Conference on Language Documentation \& Conservation). University of Hawaiia, Manoa. http://hdl.handle.net/10125/41985.

Braithwaite, Ben. 2019. “Sign language endangerment and linguistic diversity.” Language 95 (1): e161-e187. https://doi.org/10.1353/lan.2019.0025.

De Meulder, Maartje, Verena Krausneker, Graham Turner, and John Bosco Conama. 2019. "Sign language communities." In The Palgrave Handbook of Minority Languages and Communities, edited by Gabrielle Hogan-Brun and Bernadette O’Rourke, 207-232. London: Palgrave Macmillan UK.

De Vos, Connie. 2012. "Sign-spatiality in Kata Kolok: how a village sign language of Bali inscribes its signing space.” Doctoral dissertation, Max Planck Institute for Psycholinguistics.

De Vos, Connie, and Victoria Nyst. 2018. "Introduction: The time depth and typology of rural sign languages.” Sign Language Studies 18 (4): 477-487.

De Vos, Connie, and Roland Pfau. 2015. "Sign language typology: The contribution of rural sign languages.” Annual Review of Linguistics 1, no. 1 (January): 265-288. https: / / doi. org/10.1146/annurev-linguist-030514-124958.

De Vos, Connie, and Ulrike Zeshan. 2012. "Introduction: Demographic, sociocultural and linguistic variation across rural signing communities." In Sign languages in village communities: Anthropological and linguistic insights, edited by Ulrike Zeshan and Connie de Vos, 2-26. Berlin: De Gruyter Mouton. 
Eckert, Penelope, and John R. Rickford, eds. 2002. Style and Sociolinguistic Variation. Cambridge University Press. https://doi.org/10.1017/CBO9780511613258.

Engberg-Pedersen, Elisabeth. 1993. Space in Danish Sign Language: The semantics and morphosyntax of the use of space in a visual language. Hamburg: Signum.

Gonzalo, M. 2014. “Orocovis tiene la mayor comunidad de sordos.” Índice: El Nuevo Día. (December 12, 2014). https: / www.indicepr.com/noticias / 2014 / 12 / 12 / news / 33235 / orocovis-tiene-la-mayor-comunidad-de-sordos/.

LeMaster, Barbara, and Leila Monaghan. 2005. "Variation in sign languages.” In A companion to linguistic anthropology, edited by Alessandro Duranti, 141-165. Wiley.

Martínez-Cora, Marina. 2018. "Mouthings in Puerto Rican Sign Language: A closer look into language contact with spanish.” 22nd Biennial Conference of the Society for Caribbean Linguistics "Connecting the Caribbean: Languages, Borders and Identities. Heredia and Limon, Costa Rica.

2019. “Marcadores no manuales en la lengua de señas de Puerto Rico.” MA thesis, University of Puerto Rico-Río Piedras.

Nyst, Victoria. 2007. A descriptive analysis of Adamorobe Sign Language (Ghana). Utrecht: LOT.

2012. “Shared sign languages.” In Sign language: An international handbook, edited by Roland Pfau, Markus Steinbach, and Bencie Woll, 552-574. Berlin: Mouton de Gruyter.

Quinto-Pozos, David, and Robert Adam. 2015. “Sign languages in contact.” In Sociolinguistics and deaf Communities, edited by Adam C. Schembri and Ceil Lucas, 29-60. Cambridge: Cambridge University Press.

Schuit, Joke. 2013. “Typological aspects of Inuit Sign Language.” Doctoral dissertation, University of Amsterdam.

Trovato, Sara. 2013. “A stronger reason for the right to sign languages.” Sign Language Studies 13 (3): 401-422.

Williams, Hall, and Elizabeth Parks. 2012. The Puerto Rican Deaf Community. SIL Electronic Survey Report.

Woodbury, Anthony C. n.d. "What is an endangered language? - Linguistic Society of America.” https://www.linguisticsociety.org/sites/default/files/Endangered_Languages.pdf.

Zeshan. 2010. "The significance of village sign languages for the typological study of sign languages." 10th Theoretical Issues in Sign Language Research Conference (TISLR 10). Purdue University.

Zeshan, Ulrike. 2008. "Roots, leaves and branches - The typology of sign languages.” In Sign languages: spinning and unraveling the past, present and future, edited by Ronice Müller de Quadros, 671-695. Petrópolis: Editora Arara Azul.

Zeshan, Ulrike, and Connie de Vos, eds. 2012. Sign languages in village communities: Anthropological and linguistic insights. Berlin: De Gruyter Mouton. 